\title{
Escala de conexão com a natureza: evidências psicométricas no contexto brasileiro
}

\author{
Connectedness to nature scale: Psychometric \\ evidence in Brazil
}

\author{
Viviany Silva PESSOA ${ }^{1}$ \\ Valdiney Veloso GOUVEIA ${ }^{2}$ \\ Ana Karla Silva SOARES ${ }^{2}$ \\ Roosevelt VILAR ${ }^{2}$ \\ Leogildo Alves FREIRES ${ }^{3}$
}

\begin{abstract}
Resumo
Este artigo objetivou reunir evidências de validade e precisão da Connectedness to Nature Scale no contexto brasileiro. Dois estudos foram realizados. No Estudo 1, participaram 204 pessoas com idade média de 33 anos (Desvio-Padrão = 13,83), as quais responderam a referida escala e perguntas demográficas. Os resultados de uma análise de componentes principais indicaram uma estrutura unifatorial $(\alpha=0,81)$. No Estudo 2, participaram 220 estudantes universitários com idade média de 23 anos (Desvio-Padrão = 5,82), os quais responderam a escala adaptada e perguntas demográficas. Uma análise fatorial confirmatória (estimador ML) confirmou a adequação da estrutura unifatorial (e.g., Comparative Fit Index $=0,91$, Root-Mean-Square Error of Approximation $=0,064)$, obtendo consistência interna satisfatória $(\alpha=0,83)$. Concluindo, a Connectedness to Nature Scale mostrou ser uma medida psicometricamente adequada para avaliar um fator geral de conexão com a natureza, podendo ser empregada em estudos futuros.
\end{abstract}

Palavras-chave: Análise psicométrica; Conexão com a natureza; Validade fatorial.

\begin{abstract}
This article aimed to gather evidence of validity and reliability of the Connectedness to Nature Scale in Brazil. Two studies were conducted. A total of 204 people of mean age 33 years (Standard Deviation $=13.83$ ) participated in Study 1 and responded to the demographic questions and Connectedness to Nature Scale items. The results of the principal component analysis showed a one-factor structure $(\alpha=0.81)$. Two hundred and twenty undergraduate

\footnotetext{
1 Universidade Federal da Paraíba, Centro de Educação, Departamento de Psicopedagogia. João Pessoa, PB, Brasil.

2 Universidade Federal da Paraíba, Centro de Ciências Humanas, Letras e Artes, Departamento de Psicologia. Cidade Universitária, s/n., 58051-900, João Pessoa, PB, Brasil. Correspondência para/Correspondence to: V.V. GOUVEIA. E-mail: <vvgouveia@gmail.com>.

${ }^{3}$ Universidade Federal de Roraima, Centro de Educação, Departamento de Psicologia. Boa Vista, RR, Brasil. Apoio: Coordenação de Aperfeiçoamento de Pessoal de Nível Superior e Conselho Nacional de Desenvolvimento Científico e Tecnológico. Agradecimentos: À Coordenação de Aperfeiçoamento de Pessoal de Nível Superior e ao Conselho Nacional de Desenvolvimento Científico e Tecnológico pelas bolsas de doutorado e produtividade concedidas ao primeiro e segundo autores, respectivamente.
} 
students of mean age 23 years (Standard Deviation $=5.82$ ) participated in Study 2 and responded to demographic questions; an adapted version of the Connectedness to Nature Scale was used. Confirmatory factor analysis (ML estimation) confirmed the adequacy of the one-factor structure (Comparative Fit Index $=0.91$, Root-Mean-Square Error of Approximation $=0.064)$, indicating satisfactory internal consistency $(\alpha=0.83)$. In conclusion, the Connectedness to Nature Scale proved to be a psychometrically appropriate measure to assess general connectedness to nature, and it can therefore be used in future studies.

Keywords: Psychometric analysis; Connectedness to nature; Factorial validity.

A intensificação de estudos sobre comportamentos frente ao meio ambiente ocorrida na década de 1970 permitiu compreender que algumas pessoas desenvolvem afinidade com ambientes naturais, enquanto outras se sentem mais conectadas, isto é, mais "à vontade" em ambientes artificiais ou construídos (Schultz, Shriver, Tabanico, \& Khazian, 2004). Essas diferenças individuais para a identificação e o posicionamento frente ao ambiente, seja ele natural ou construído, têm implicações em atitudes e comportamentos pró-ambientais e, consequentemente, na qualidade de vida das gerações presentes e futuras.

Psicólogos têm sido motivados a entender e explicar os aspectos estruturantes desse tipo de identificação, assim como a destacar os elementos que interferem no nível de contato entre pessoa e natureza. Inicialmente, as pesquisas evidenciavam construtos como conhecimento, atitudes e preocupação ambiental (Maloney \& Ward, 1973). Entretanto, com o desenvolvimento da temática, conteúdos afetivos também começaram a ser levados em consideração para explicar os comportamentos voltados para a natureza e seus recursos.

Nesta direção, estudos baseados em argumentos de ecólogos e ecopsicólogos começaram a enfatizar o afeto como componente para o entendimento desse tipo de comportamento (Schultz, 2002). Esse desenvolvimento ratificou a necessidade de pesquisas sobre a conexão com a natureza, definida por Mayer e Frantz (2004) como um traço individual que corresponde ao sentimento de conexão emocional com o mundo natural, ou seja, que indica o quanto o indivíduo sente que faz parte desse mundo.

Estudos apontam que essa conexão, além de indicar relações substanciais com outros aspectos 272 psicossociais importantes para a conduta humana, como o bem-estar subjetivo (Mayer \& Frantz, 2004) e os valores humanos (Pessoa, 2011), é capaz de incentivar e auxiliar na manutenção do padrão comportamental frente à natureza e seus recursos (Gosling \& Williams, 2010; Schultz, 2002). Dessa forma, é possível considerar a conexão com a natureza como uma temática útil em estudos interessados na explicação e na promoção de uma relação saudável entre o ser humano e o meio ambiente.

Considerando a importância dessa conexão para o entendimento de comportamentos que afetam o ambiente, explorar esse construto é um passo importante para entender como as pessoas se relacionam com a natureza e como agem sobre ela. Para tanto, especialistas na temática têm desenvolvido métodos e medidas apropriados para a observação e avaliação da conexão com a natureza (Cervinka, Röderer, \& Hefler, 2012; Mayer \& Frantz, 2004; Schultz, 2001, 2002), o que é um passo fundamental no sentido de estimar o quanto as pessoas estão envolvidas e em que medida isso pode repercutir em suas vidas.

\section{Panorama dos estudos na área}

É preciso destacar que do ponto de vista da Psicologia a conexão com a natureza pode ser entendida a partir de três dimensões: cognitiva, afetiva e comportamental (Schultz, 2002). Embora a diferença entre cada uma seja tênue, faz-se necessário considerar suas particularidades. A dimensão cognitiva destaca a representação do self frente à natureza, indicando o quanto o indivíduo acredita que é parte dela ou o quanto percebe que ela é parte de si (Schultz, 2001, 2002). A dimensão afetiva destaca o sentimento de intimidade, familiaridade e aproximação com a natureza. O incremento de origem emocional, por sua vez, favorece a busca por 
conhecimento e cuidado para com o elemento de relação (Mayer \& Frantz, 2004); estudos que destacam esse aspecto sugerem a contribuição deste para o entendimento de atitudes e comportamentos relacionados (Kals, Schumacher, \& Montada, 1999). Por fim, a dimensão comportamental expressa o nível de comprometimento das pessoas frente à natureza, uma vez que considera a ação em prol da mesma uma resultante de crenças e intenção de cuidado.

Mesmo sendo considerada em função de dimensões, a conexão com a natureza, de uma perspectiva psicológica, precisa ser entendida, avaliada e explicada como um fenômeno complexo capaz de auxiliar na compreensão de comportamentos frente aos mais diferentes elementos do ambiente natural. Esse fato torna necessário o desenvolvimento de medidas de relação pessoaambiente natural que reúnam evidências de validade e consistência interna, sendo adequadas para o contexto em análise e seguindo a proposta mais atual para o processo de validação de instrumentos (American Educational Research Association, American Psychological Association, \& National Council on Measurement in Education, 1999; Primi, Muniz, \& Nunes, 2009).

De fato, a literatura conta com várias medidas para avaliar esse tema. A propósito, Hefler, Zeidler e Cervinka (2009) fizeram uma compilação de escalas de conexão com a natureza, relatando a existência de dez opções de medidas (Coelho, 2009); também é possível encontrar testes de associação implícita (Bruni \& Schultz, 2010; Schultz et al., 2004) e três outras medidas relacionadas ao construto (Dunlap \& van Liere, 1978; Ellis \& Thompson, 1997; Kaiser \& Wilson, 2004; Schultz, 2000).

Certamente, cada uma das medidas anteriormente encontradas apresentaram contribuições para a temática. No entanto, não foi possível identificar nenhuma medida adaptada ao contexto brasileiro. Neste sentido, com a intenção de ampliar os estudos sobre conexão com a natureza e oferecer a opção de um instrumento adequado para avaliála, optou-se por conhecer evidências de validade com base na estrutura interna e de precisão (consistência interna) da Connectedness to Nature Scale (CNS, Escala de Conexão com a Natureza) (Mayer \& Frantz, 2004).

\section{Escala de Conexão com a Natureza}

Criada por Mayer e Frantz (2004), a Escala de Conexão com a Natureza (ECN) é uma medida que visa avaliar o quanto a pessoa se sente integrada e conectada ao ambiente a partir de uma perspectiva afetiva e individual. Ela é composta por um conjunto de 14 itens os quais devem ser respondidos em uma escala de 5 pontos, variando de 1 (Discordo totalmente) a 5 (Concordo totalmente). Ao assumirem uma rotação não ortogonal, considerando os critérios de Kaiser (eigenvalue) e Cattell (scree plot) e sem especificar o método de extração, as evidências de validade interna da medida original indicaram uma estrutura unifatorial, apresentando cargas fatoriais médias de 0,61, correspondendo a valor próprio de 5,29, que foi responsável por explicar 38\% da variância total. Seus índices de precisão (consistência interna) foram considerados satisfatórios, apresentando alfa de Cronbach de 0,84 e teste-reteste de 0,82. Essa medida correlacionou-se positivamente com comportamento ecológico, ambientalismo, satisfação com a vida, orientação valorativa geral, tomada de perspectiva disposicional e tomada de perspectiva ambiental. Portanto, apresentou evidências de validade concorrente.

Olivos, Aragonés e Amérigo (2011) procederam à adaptação da referida medida para o contexto espanhol, considerando duas amostras (estudantes e população geral). Esses autores empregaram a versão original de 14 itens, mas observaram que o item 12 (Cuando pienso en mi lugar en la Tierra, me considero a mi mismo como miembro de la cúspide jerárquica que existe en la naturaleza) apresentou carga fatorial inferior a 0,20. Por conta disso, optaram por excluí-lo e procederam com uma nova análise considerando apenas 13 itens. Os resultados indicaram índices satisfatórios de consistência interna (alfa de Cronbach): 0,76 (estudantes) e 0,74 (população geral). De acordo com Olivos et al. (2011), verificou-se uma coerência entre os achados da pesquisa realizada por eles com aqueles da pesquisa original. Na mesma direção, Cervinka et al. (2012) relataram que a ECN foi adaptada ao contexto alemão com bons indicadores 
de adequação psicométrica, considerado sua versão de 13 itens (reportaram, por exemplo, alfa de Cronbach de 0,85).

Embora Perrin e Benassi (2009) argumentem que a medida de conexão com a natureza apresente itens característicos de uma medida cognitiva (e.g., "Penso na natureza como uma comunidade da qual faço parte"; "Quando penso sobre meu lugar na Terra, me considero no topo da hierarquia que existe na natureza", etc.), eles afirmam que ela apreende o sentimento de conexão com a natureza dos respondentes. Em contexto estadunidense, esses autores também corroboraram a adequação de sua estrutura unifatorial por meio de análises fatoriais exploratória e confirmatória, com uma consistência interna de 0,75.

Como é possível observar, a ECN, independentemente do contexto cultural, vem se mostrando um instrumento psicometricamente adequado, tendo a vantagem de focar no componente afetivo, resolvendo limitações identificadas em medidas prévias que são mais comumente utilizadas, como a Inclusion of Nature in Self Scale (Schultz, 2001) e a Implicit Association Test-Nature Scale (Schultz et al., 2004). Nesse sentido, a ECN é promissora, compondo-se de um conjunto de itens de fácil compreensão e resposta. Assim, é justificada a necessidade do seu estudo para a realidade brasileira. Nessa direção, o objetivo deste artigo foi reunir evidências de validade com base na estrutura interna (Primi et al., 2009) e de precisão (consistência interna) da Connectedness to Nature Scale (Mayer \& Frantz, 2004), favorecendo seu uso no Brasil.

\section{Estudo 1. Análise exploratória da Escala de Conexão com a Natureza}

Este estudo descreve a análise preliminar da ECN. Desta forma, objetiva reunir as primeiras evidências psicométricas acerca de sua validade fatorial e consistência interna. Vale salientar que essa medida foi originalmente proposta como sendo unifatorial (Mayer \& Frantz, 2004). Procurou-se, assim, conhecer se as evidências empíricas poderiam

274 apoiar essa estrutura.

\section{Método}

\section{Participantes}

Participaram 204 pessoas de uma capital nordestina, com idades variando de 16 a 71 anos (Média - $M=33,10$; Desvio-Padrão - $D P=13,83$ ), a maioria do sexo feminino $(60,3 \%)$, solteira $(57,4 \%)$ e de classe média (59,6\%). Tratou-se de uma amostra de conveniência (não probabilística), fazendo parte aquelas pessoas que, sendo solicitadas a colaborar, concordaram em fazê-lo.

\section{Instrumentos}

Os participantes responderam um questionário composto de duas partes:

- Escala de Conexão com a Natureza (ECN): criada por Mayer e Frantz (2004), tendo como objetivo verificar o aspecto afetivo da relação pessoa-ambiente. É composta por 14 itens (e.g., "Penso na natureza como uma comunidade da qual faço parte"; "Meu bem-estar pessoal independe do bem-estar da natureza", etc.), os quais são respondidos em escala de 5 pontos, variando de 1 (Discordo totalmente) a 5 (Concordo totalmente). Seus parâmetros psicométricos foram discutidos previamente.

- Perguntas demográficas: estas tinham o intuito de caracterizar os participantes do estudo, incluindo informações sobre sexo, idade, ocupação, nível econômico, disponibilidade para pagar por energia de fontes renováveis, frequência de atividade de cuidado ambiental e disponibilidade para participar de projetos pró-ambientais.

\section{Procedimentos}

Como a Escala de Conexão com a Natureza foi originalmente elaborada em língua inglesa, não tendo sido encontrada qualquer versão em português, foi necessário traduzi-la. No caso, recorreu-se ao procedimento de back translation (retrotradução), contando com a colaboração de dois psicó- 
logos brasileiros com vivências nos contextos britânico e neozelandês, ambos especialistas em temas sociais e ambientais. Um deles traduziu o instrumento do inglês para o português e o outro, considerando essa última versão, voltou a traduzi-lo para a língua original. Depois, as duas versões em inglês foram comparadas. Como não foram observadas variações substanciais, definiu-se a versão em português da ECN, a qual poderá ser obtida através do contato com qualquer um dos autores deste artigo.

Na sequência, a coleta de dados foi realizada em diversos contextos (e.g., sala de aula, residência etc.), primando-se pelas respostas individuais dos participantes. No momento da aplicação, os colaboradores descreviam como responder o questionário, enfatizando o caráter voluntário da participação e assegurando o anonimato dos respondentes. Uma vez tendo concordado com a participação no estudo, o indivíduo assinava o Termo de Consentimento Livre e Esclarecido, previsto no projeto que obteve parecer favorável do Comitê de Ética em Pesquisa com Seres Humanos (Processo nº 0028.0.126.000-11). Em média, dez minutos foram suficientes para concluir a participação no estudo.

O software Statistical Package for the Social Sciences (SPSS, versão 18) foi empregado para a tabulação e análise dos dados, permitindo calcular estatísticas descritivas (frequência, média, desvio-padrão), teste $t$ de Student (poder discriminativo dos itens), correlação item-total (homogeneidade do fator), análise dos componentes principais (estrutura fatorial) e alfa de Cronbach (consistência interna). Com o programa estatístico $R$ (R Development Core Team, 2011; van der Ark, 2007) foi realizada a Análise Mokken, uma análise não paramétrica baseada na Teoria de Resposta ao Item (TRI), utilizada para comprovar a unidimensionalidade da medida verificando os pressupostos de homogeneidade monotônica e de monotonicidade dupla (Mokken \& Lewis, 1982). Para tanto, têm-se em conta os índices de escalabilidade $H$ de Löevinger ( $H$ para a escala total e $H s$ para cada item), considerando-se aceitáveis valores acima de 0,30, e Rho de Mokken, com valores ideais acima de 0,80.

\section{Resultados}

Inicialmente, foram invertidas as escalas de resposta de três itens que apresentavam semântica contrária à maioria daqueles que compunham a escala (itens 4 [Meu bem-estar pessoal independe do bem-estar da natureza], 7 [Frequentemente me sinto desconectado/a da natureza] e 14 [Quando penso sobre meu lugar na Terra, me considero no topo da hierarquia que existe na natureza]). $\mathrm{Na}$ sequência, a somatória dos 14 itens resultou em uma pontuação total da qual foi calculada a mediana $(M d=54)$, considerada para criar os grupos inferior e superior. Por fim, para cada item, empregou-se o teste $t$ de Student a fim de comparar as médias das pessoas dos dois grupos, tendo todos os itens apresentado poder discriminativo satisfatório $(t>3, p<0,001)$.

Observando-se a adequação dos itens para discriminar os participantes, seguiu-se com as demais análises estatísticas. No caso, procurou-se, primeiramente, checar a adequação da realização de uma análise fatorial com a matriz de correlação inter-itens, sendo que a mesma se mostrou adequada (Kaiser-Meyer-Olkin [KMO] $=0,84$ e Teste de Esfericidade de Bartlett, $\chi^{2}(91)=664,03, p<0,001$ ). Na ocasião, decidiu-se efetuar uma análise de componentes principais, método mais indicado quando o foco é a redução de fatores naqueles que explicam a máxima variância (Hair, Black, Babin, Anderson, \& Tatham, 2009), porém sem fixar tipo de rotação ou número de fatores a extrair. Considerando o critério de Kaiser (eigenvalue ou valor próprio igual ou superior a 1), observou-se a possibilidade de extrair quatro componentes, cujos valores próprios foram 4,38, 1,22, 1,17 e 1,00, explicando conjuntamente 55,5\% da variância total. Não obstante, a distribuição (scree test) desses eigenvalues (critério de (attell) sugeriu uma estrutura unifatorial.

No entanto, com o fim de dirimir dúvidas, realizou-se uma análise paralela (critério de Horn), assumindo os parâmetros do banco de dados (204 participantes e 14 variáveis), efetuando 1 mil simulações. Esta gerou um segundo valor próprio simulado de 1,46, superando o observado, o que corrobora a estrutura unidimensional da medida. Portanto, fixou-se a extração de um único com- 
ponente, cujos resultados são apresentados na Tabela 1.

De acordo com essa tabela, parece adequado pensar na ECN como uma medida unidimensional, tendo seu componente geral apresentado eigenvalue de 4,38, explicando 31,6\% da variância total. Em geral, seus itens mostraram saturações adequadas, situando-se acima de $|0,30|$. No entanto, o item 14 ("Quando penso sobre meu lugar na Terra, me considero no topo da hierarquia que existe na natureza") apresentou carga fatorial baixa $(0,17)$, o que sugere a possibilidade de eliminá-lo. Vale ressaltar que a análise da escalabilidade de Mokken apresentou resultados limítrofes $(H=0,26$ e $R h o=0,81 ; H s$ variando entre 0,09 [item 14] e 0,36 [item 10]), o que pode indicar evidência não tão robusta da unidimensionalidade dessa medida.

Contrapondo o resultado anteriormente descrito, os achados referentes aos indicadores de homogeneidade e consistência interna parecem mais animadores, reforçando o que se observou na análise de componentes principais. Especificamente, foram constatadas correlações médias inter-itens de 0,27 e item-total de 0,47, sendo o alfa de Cronbach de 0,82 . Não foi encontrada diferença estatisticamente significativa quando o valor do alfa foi comparado com os verificados no estudo original (Mayer \& Frantz, 2004), estudo $1 \alpha=0,84, \mathrm{n} \leq 60$ $\left(M_{H-W}<1\right)$ e teste-reteste $\alpha=0,82, \mathrm{n} \leq 65\left(M_{H-W}<1\right)$. No entanto, ao comparar com a pesquisa de Perrin e Benassi (2009) $\alpha=0,75, \mathrm{n} \leq 361\left(M_{H-W}=0,66\right.$, $p=0,04)$ e as de Olivos et al. (2011), com participantes da população geral $\alpha=0,70, \mathrm{n} \leq 112$ $\left(M_{H-W}=8,15, p=0,02\right)$, verificou-se que são estatisticamente diferentes.

\section{Discussão parcial}

De forma geral, as análises adotadas neste estudo demonstraram que a estrutura unidimensional pode ser considerada coerente com a proposta original da medida (Mayer \& Frantz, 2004) e

Tabela 1

Estrutura fatorial da Escala de Conexão com a Natureza

\begin{tabular}{|c|c|c|}
\hline Itens & Conteúdo dos itens & Saturação \\
\hline 12 & Com frequência, sinto-me parte da teia da vida. & 0,75 \\
\hline 11 & Sinto, com frequência, uma identificação com animais e plantas. & 0,69 \\
\hline 10 & Sinto que todos os habitantes da Terra, humanos e não humanos, compartilham uma força vital comum. & 0,66 \\
\hline 06 & Da mesma forma que uma árvore pode fazer parte da floresta, me sinto parte da natureza como um todo. & 0,65 \\
\hline 02 & Penso na natureza como uma comunidade da qual faço parte. & 0,63 \\
\hline 08 & Muitas vezes sinto uma sensação de união com a natureza ao meu redor. & 0,62 \\
\hline 05 & Quando penso em minha vida, me vejo como parte de um amplo processo cíclico de existência. & 0,58 \\
\hline 09 & Reconheço e aprecio a inteligência de outros seres vivos. & 0,54 \\
\hline 13 & Tenho um entendimento claro de como minhas ações afetam a natureza. & 0,50 \\
\hline 03 & Tenho a sensação de que pertenço à Terra da mesma forma que ela me pertence. & 0,50 \\
\hline 07 & Frequentemente me sinto desconectado(a) da natureza. (R) & 0,45 \\
\hline 01 & $\begin{array}{l}\text { Muitas vezes sinto que sou apenas uma pequena parte da natureza ao meu redor, e que não sou mais importante que a } \\
\text { grama no chão ou os pássaros. }\end{array}$ & 0,42 \\
\hline 04 & Meu bem-estar pessoal independe do bem-estar da natureza. (R) & 0,39 \\
\hline 14 & Quando penso sobre meu lugar na Terra, me considero no topo da hierarquia que existe na natureza. (R) & 0,17 \\
\hline \multicolumn{2}{|c|}{ Número de itens } & 14,00 \\
\hline \multicolumn{2}{|c|}{ Valor Próprio } & 4,38 \\
\hline \multicolumn{2}{|c|}{ \% da variância } & 31,60 \\
\hline \multicolumn{2}{|c|}{ Alfa de Cronbach $(\alpha)$} & 0,82 \\
\hline
\end{tabular}

Nota: R: Item cuja escala de resposta foi invertida. 
com estudos anteriores os quais adaptaram a ECN para outros contextos socioculturais (Olivos et al., 2011; Perrin \& Benassi, 2009). No entanto, ao comparar os coeficientes de consistência interna de pesquisas anteriores foi verificado que alguns se diferenciaram estatisticamente dos do presente estudo, o que pode sugerir uma flutuação amostral e, ainda, alguma especificidade que sinaliza a oportunidade para mais pesquisas (Hakstian \& Whalen, 1976; Kim \& Feldt, 2008).

Verificou-se, também, uma possível melhora nos indicadores psicométricos dessa medida, quando considerada uma versão sem o item 14 ("Quando penso sobre meu lugar na Terra, me considero no topo da hierarquia que existe na natureza"), o mesmo item excluído na versão adaptada para o contexto espanhol (Olivos et al., 2011). Portanto, decidiu-se ter em conta essa nova versão em um segundo estudo com o objetivo de testá-la por meio de análises estatísticas mais robustas, como descrito a seguir.

\section{Estudo 2. Confirmação da estrutura fatorial da Escala de Conexão com a Natureza}

O Estudo 1 apresentou evidências de uma estrutura unifatorial. No entanto, os resultados do critério de Kaiser, da análise da escalabilidade de Mokken e a baixa saturação de um dos seus itens (Item 14) motivou a realização deste segundo estudo. Nele, buscou-se apresentar evidências mais robustas (análise fatorial confirmatória) da adequação da estrutura unifatorial da ECN eliminando-se o item 14.

\section{Método}

\section{Participantes}

Participaram 220 estudantes universitários, com idade média de 23 anos ( $D P=5,82$, amplitude de 17 e 48 anos), a maioria do sexo feminino (60,6\%). Tratou-se de uma amostra de conveniência (não probabilística), tendo participado as pessoas que, presentes em sala de aula e convidadas a colaborar, concordaram em fazê-lo voluntariamente.

\section{Instrumentos e Procedimentos}

Os participantes responderam o mesmo questionário apresentado no Estudo 1, o qual reunia a ECN e perguntas demográficas. Não obstante, no presente estudo, o item 14 da ECN foi eliminado, justificando-se em razão de sua baixa saturação na pesquisa anterior. Adotou-se a mesma escala de resposta previamente descrita. A coleta dos dados foi realizada em contexto coletivo de sala de aula, porém as respostas foram dadas individualmente. Esta pesquisa integrou o projeto submetido ao Comitê de Ética citado anteriormente, tendo sido aprovada. Em média, os estudantes concluíram sua participação em dez minutos.

O software Amos (versão 18) foi utilizado para realização da Análise Fatorial Confirmatória (AFC), objetivando comprovar a estrutura unifatorial da versão adaptada da ECN, composta por 13 itens. Para tanto, considerou-se a matriz de covariância como entrada, tendo sido adotado o método de estimação Maximum Likelihood (ML). Com o fim de verificar a qualidade de ajuste do modelo testado, foram levados em consideração múltiplos indicadores de ajuste comumente empregados na literatura (Byrne, 2010; Hair et al., 2009; Hooper, Coughlan, \& Mullen, 2008; Tabachnick \& Fidell, 2013):

- $\chi^{2}$ (Qui-quadrado): avalia a probabilidade do modelo teórico se ajustar aos dados. Nele, valores baixos são desejáveis. Contudo, em razão de ser influenciado pelo tamanho da amostra e o número de variáveis do modelo, costuma-se ter em conta sua razão quanto aos graus de liberdade (Razão $\left.\chi^{2} / \mathrm{gl}\right)$. Neste caso, um modelo ajustado apresenta valores entre 2 e 3 , porém aceita-se até 5 como indicador de adequação do modelo teórico.

- GFI (Goodness-of-Fit Index) e AGFI (Adjusted Goodness-of-Fit Index): expressam a proporção de variância-covariância nos dados explicada pelo modelo, sendo que o AGFI é ponderado em relação aos seus graus de liberdade. Seus valores variam de 
0 a 1, aceitando-se como indicadores de ajuste do modelo aqueles próximos ou superiores a 0,90.

- CFI (Comparative Fit Index): funciona satisfatoriamente quando o tamanho da amostra é pequeno (Tabachnick \& Fidell, 2013). É um indicador comparativo adicional de ajuste ao modelo, sendo que valores próximos ou superiores a 0,90 sugerem um melhor ajuste do mesmo.

- RMSEA (Root-Mean-Square Error of Approximation): um dos indicadores de ajuste mais informativo e parcimonioso, pois seleciona o modelo com menor número de parâmetros. Recomendam-se valores entre 0,05 e 0,08, admitindo-se até 0,10 como expressando um ajuste satisfatório.

\section{Resultados}

Fixando que os 13 itens da ECN saturassem em um único fator, obtiveram-se os indicadores de ajuste a seguir: $\chi^{2}(65)=164,56, p<0,001$, $\chi^{2} / \mathrm{gl}=2,53, \mathrm{GFI}=0,89, \mathrm{AGFI}=0,85, \mathrm{CFI}=0,78 \mathrm{e}$ RMSEA $=0,084$ (Intervalo de Confiança - IC90\% = 0,068 - 0,100). Entretanto, levando em consideração os Modification Indices (MI), percebeu-se que o modelo com erros correla-cionados incrementou os índices de ajuste (Brown, 2003), o que justificou fixar a correlação entre os pares de parâmetros de erros referentes aos itens 7 (Frequentemente me sinto desconectado(a) da natureza) e 8 (Muitas vezes sinto uma sensação de união com a natureza ao meu redor) ( $\mathrm{Ml}=25,05)$. Os resultados dessa análise são sumarizados na Figura 1.

Os indicadores de ajuste para esse modelo reespecificado foram, em seu conjunto, mais promissores, atendendo às recomendações da literatura de modo satisfatório: $\chi^{2}(64)=137,40, \chi^{2} / g l=2,15$, $\mathrm{GFI}=0,91, \mathrm{AGFI}=0,87, \mathrm{CFI}=0,84$ e RMSEA $=$ $0,073($ IC90\% = 0,056 - 0,089). É importante destacar que todos os pesos fatoriais (Lambdas $-\lambda$ ) foram estatisticamente diferentes de zero $(z>1,96, p<0,05)$, variando entre 0,22 (Item 4, "Meu bem-estar pessoal independe do bem-estar da natureza") e 0,65 (Item 12, "Com frequência, sinto-me parte da teia

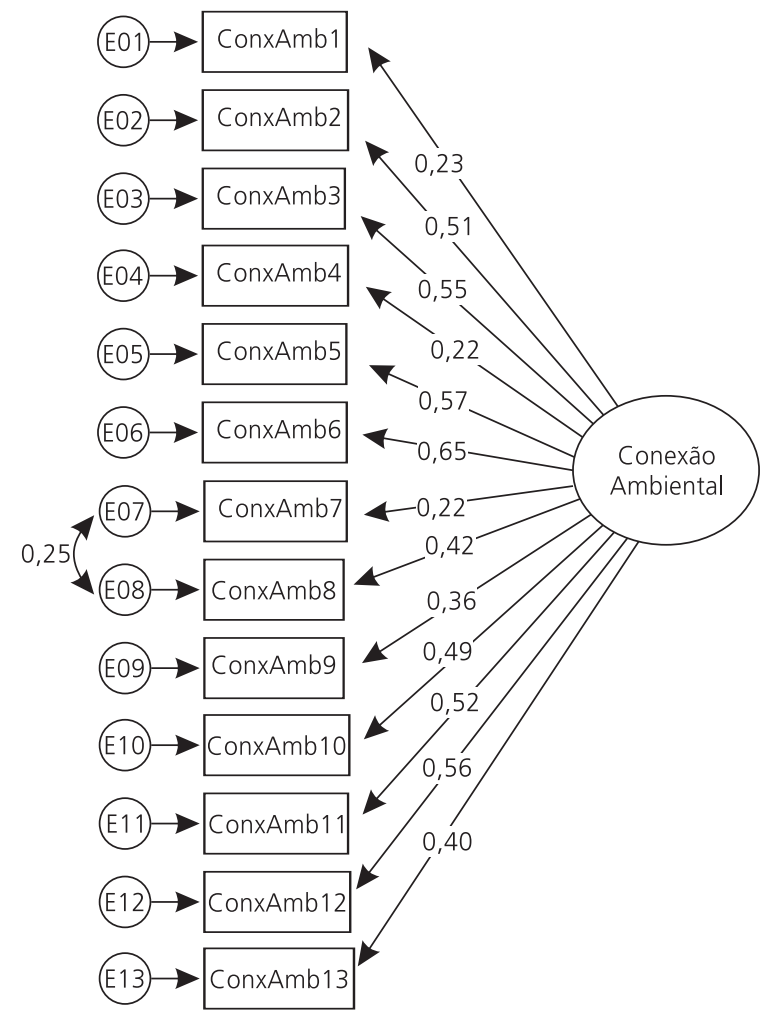

Figura 1. Estrutura fatorial da Escala de Conexão com a Natureza. Nota: ConxAmb: ConexãoAmbiental.

Por fim, checaram-se os indicadores de homogeneidade e consistência interna dessa medida. Especificamente, foram observadas correlações médias inter-itens $(0,27)$ e item-total $(0,47)$ as quais revelaram uma medida homogênea, tendo sido seu alfa de Cronbach de 0,83 , refletindo padrão desejável de consistência interna. Quando esse coeficiente foi comparado com o do Estudo 1, observou-se que não houve diferença significativa $\left(M_{H-W}<1\right.$; Hakstian \& Whalen, 1976), endossando a robustez do achado.

\section{Discussão parcial}

Com base nas análises de natureza confirmatória previamente descritas, foi possível admitir uma adequação de ajuste do modelo para a versão de 13 itens da ECN. Embora os indicadores de ajuste $\operatorname{AGFI}(0,87)$ e CFI $(0,84)$ possuam valores razoáveis, quando analisados em conjunto com os demais 
indicadores apoiam a adequação psicométrica da estrutura sugerida, ou seja, reúnem evidências de validade fatorial e consistência interna.

Além disso, tomando como base Brown (2003), é possível admitir que os erros correlacionados podem representar tanto itens com semânticas semelhantes, como eventuais dificuldades de leitura de itens invertidos e até particularidades de demanda. $E$, mesmo que a proximidade semântica não seja suficiente para explicar as correlações, é possível assumir que alguma relação não especificada pode influenciar essa associação.

Deste modo, configura-se como uma opção para conhecer os correlatos da conexão com a natureza. De fato, a versão reduzida é vista como pertinente, uma vez que preserva a estrutura original e descarta um item que já tinha se revelado pouco adequado em estudos prévios (Cervinka et al., 2012; Olivos et al., 2011).

\section{Discussão Geral}

As duas pesquisas apresentadas atenderam ao objetivo principal de reunir evidências de validade baseadas na estrutura interna e de precisão (consistência interna) da ECN (Mayer \& Frantz, 2004) para o contexto brasileiro. Entretanto, anteriormente, também foram oferecidas informações sobre a qualidade de seus itens. Isso permite agregar informações acerca das qualidades dessa medida, oferecendo evidências psicometricamente adequadas para sua utilização em estudos futuros. Discutem-se a seguir os principais resultados.

Com o fim de conhecer a discriminação dos itens da ECN, recorreu-se ao critério mais robusto da mediana (Pasquali, 2003), observando que todos os itens dessa escala apresentaram capacidade métrica de diferenciar os participantes que tiveram magnitudes próximas no traço latente avaliado (conexão com a natureza). Deste modo, individualmente, os itens parecem funcionar como esperado; fez-se necessário, entretanto, conhecer como se comportam no conjunto, avaliando as propriedades de validade fatorial e consistência interna.
Nessa direção, recorreu-se a múltiplos critérios (e.g., eigenvalue, análise paralela, análise confirmatória) para conhecer a solução fatorial dessa medida. Deste modo, a estrutura fatorial da ECN foi testada em dois estudos independentes, sendo que um adotou um procedimento exploratório (análise de componentes principais) e o outro, confirmatório (analise fatorial confirmatória). No primeiro caso, coerente com o estudo original de Mayer e Frantz (2004), constatou-se que o fator geral emergiu espontaneamente, isto é, sem fixar número de fatores a extrair ou tipo de rotação, corroborando os achados quando critérios robustos de extração de fatores são calculados, como no caso da análise paralela (Hayton, Allen, \& Scarpello, 2004)

Entretanto, assim como o encontrado por Cervinka et al. (2012) e Olivos et al. (2011), na presente pesquisa um dos 14 itens não apresentou saturação adequada ( $H>0,30$; Tabachnick \& Fidell, 2013), resultando em uma estrutura unidimensional composta por 13 itens. Esses resultados foram confirmados quando uma análise fatorial confirmatória foi realizada, observando-se indicadores de ajuste que, mesmo não sendo perfeitos, aproximaram-se daqueles preconizados na literatura como sendo satisfatórios (Hair et al., 2009; Tabachnick \& Fidell, 2013).

Quanto à consistência interna da medida, a versão brasileira adaptada reuniu indicadores os quais suportam sua evidência. A propósito, os alfas de Cronbach observados nos dois estudos estavam acima do ponto de corte comumente recomendado na literatura (0,70; Pasquali, 2003). Reforçando a estabilidade desse parâmetro, quando comparados os coeficientes dos dois estudos, estes não diferiram estatisticamente $\left(M_{\mathrm{H}-\mathrm{W}}<1\right.$; Kim \& Feldt, 2008). Na mesma direção, comprovaram-se correlações interitens (indicador de homogeneidade) superiores a comumente recomendada $(0,20 ;$ Clark \& Watson, 1995). Em suma, parece claro, pois, que essa medida apresenta evidências de consistência interna, consoante com o que já havia sido encontrado na literatura (Cervinka et al., 2012; Mayer \& Frantz, 2004; Olivos et al., 2011). 
Apesar dos achados animadores que recomendam a utilização da presente medida no âmbito da realidade brasileira, os estudos aqui descritos não estão isentos de limitações, a exemplo das amostras consideradas. Não se deve perder de vista que as mesmas foram muito específicas, considerando, sobretudo, os estudantes. Isso certamente limita a expansão das conclusões para além de seu escopo, limitado a grupos específicos de uma capital do Nordeste brasileiro considerados por conveniência. Todavia, também é importante salientar que o propósito deste artigo não foi estabelecer quaisquer relações do construto Conexão com a Natureza com variáveis outras, mas reunir evidências de validade baseadas na estrutura interna e na consistência interna de uma medida específica. Assim, as amostras com 200 participantes ou mais parecem atender plenamente às exigências para levar a cabo análises estatísticas robustas as quais atestem a qualidade psicométrica da versão abreviada da escala (Pasquali, 2003).

No entanto, amostras mais amplas e diversificadas devem ser levadas em consideração no futuro, incluindo pessoas de níveis escolares e econômicos variados, assim como com experiências diversas quanto ao contato real com a natureza. Além disso, é preciso pensar em reunir evidências complementares de validade, checando a correlação da ECN com construtos similares (validade convergente) e distintos (validade discriminante). De igual modo, caberá avaliar a estabilidade temporal dessa medida.

Entretanto, em razão do pensamento "politicamente correto" inerente às questões ambientais, há de se pensar, também, na possibilidade de desenvolver medidas que sejam menos afetadas pela desejabilidade social. Considerar medidas implícitas, por exemplo, poderá ser uma alternativa, como fizeram Schultz et al. (2004) e Bruni e Schultz (2010), focando na dimensão afetiva das atitudes implícitas. Porém, também podem ser realizadas pesquisas as quais busquem conhecer contextos e elementos que estão associados com bons indicadores de conexão com a natureza, e talvez se os valores que as pessoas assumem como princípios importante, sobretudo aqueles que indicam uma orientação menos materialista, como os suprapessoais (Gouveia, 2013).

\section{Colaboradores}

Todos os autores deram contribuições substanciais em todas as etapas do artigo, a saber: concepção e desenho, análise e interpretação dos dados, revisão e aprovação da versão final.

\section{Referências}

American Educational Research Association, American Psychological Association, \& National Council on Measurement in Education (1999). Standards for educational and psychological testing. Washington, DC: American Educational Research Association.

Brown, T. A. (2003). Confirmatory fator analysis of the Penn State Worry Questionnaire: Multiple factors or method effects? Behaviour Research and Therapy, 41(12), 1411-1426. Retrieved October 17, 2014, from http://sws1.bu.edu/tabrown/Manuscripts/Brown\% 202003.pdf

Bruni, C. M., \& Schultz, W. (2010). Implicit beliefs about self and nature: Evidence from an IAT game. Journal of Environmental Psychology, 30(1), 95-102. Retrieved July 27, 2012, from http://ac.els-cdn.com/S02724 94409000784/1-s2.0-50272494409000784-main. pdf?_tid=0cc8757a-5956-11 e4-8da0-00000aacb36 $1 \& a c d n a t=1413918864 \_a e 79755$ a214c30f42aec $9294 f 583 e 487$

Byrne, B. M. (2010). Structural equation modeling with Amos: Basic concepts, applications, and programming $\left(2^{\text {nd }}\right.$ ed.). New York: Routledge.

Cervinka, R., Röderer K., \& Hefler, E. (2012). Are nature lovers happy? On various indicators of well-being and connectedness with nature. Journal of Health Psychology, 17(3), 379-388. Retrieved January 29, 2013, from http://hpq.sagepub.com/content/17/3/ 379.full.pdf

Clark, L. A., \& Watson, D. (1995). Constructing validity: Basic issues in objective scale development. Psychological Assessment, 7(3), 309-319. Retrieved February 10, 2013, from http://lep.unige.ch/system/ files/issas/files/clark_watson_1995_psychass.pdf

Coelho, J. A. P. M. (2009). Habilidade de conservação de água: uma explicação pautada em valores humanos, emoções e atitudes ambientais (Tese de doutorado em não-publicada). Universidade Federal da Paraíba, João Pessoa. Recuperado em junho 28, 2013, de http:// www.vvgouveia.net/en/images/Teses/Coelho_J._A. _P._M._2009.pdf.pdf 
Dunlap, R. E., \& van Liere, K. D. (1978). The new environmental paradigm. Journal of Environmental Education, 9(4), 10-19.

Ellis, R. J., \& Thompson, F. (1997). Culture and the environment in the pacific northwest. American Political Behavior, 91(4), 885-897. Retrieved July 28, 2012, from http://papers.ssrn.com/sol3/papers.cfm? abstract_id=1123672

Gosling, E., \& Williams, K. J. H. (2010). Connectedness to nature, place attachment and conservation behavior: Testing connectedness theory among farmers. Journal of Environmental Psychology, 30(3), 298-304. Retrieved July 28, 2012, from http://ac.elscdn.com/S027249441000006X/1-s2.0-S027249 441000006Xmain.pdf?_tid=004ffc7c-5957-11e48224-00000aab0f6b\&acdnat=1413919273_3b 9160974bc 45732e61dc2df7196e318

Gouveia, V. V. (2013). Teoria funcionalista dos valores: fundamentos, aplicações e perspectivas. São Paulo: Casa do Psicólogo.

Hair Jr., J. F., Black, W. C., Babin, B. J., Anderson, R. E., \& Tatham, R. L. (2009). Análise multivariada de dados (6 $6^{\mathrm{a}}$ ed.). Porto Alegre: Bookman.

Hakstian, A. R., \& Whalen, T. E. (1976). A k-sample significance test for independent alpha coefficients. Psychometrika, 41(2), 219-231. Retrieved February 10, 2013, from http://link.springer.com/article/10.1007\% 2FBF02291840

Hayton, J. C., Allen, D. G., \& Scarpello, V. (2004). Factor retention decisions in exploratory factor analysis: A tutorial on parallel analysis. Organizational Research Methods, 7(2), 191-205. Retrieved February 15, 2013, from http://orm.sagepub.com/content/7/2/191. full. pdf+html

Hefler, E., Zeidler, D., \& Cervinka, R. (2009). Connectedness with nature and support in life through spirituality. In H. Gutscher, H. J. Mosler, B. Meyer, S. Mischke, \& M. Soland (Eds.), $8^{\text {th }}$ Biennial Conference Environmental Psychology (pp.84). Zürich: Universität Zürich. Retrieved May 12, 2012, from http://www. meduniwien.ac.at/umwelthygiene/p018_cn_ spirituality.pdf

Hooper, D., Coughlan, J., \& Mullen, M. R. (2008). Structural equation modeling: Guidelines for determining model fit. Journal of Business Research Methods, 6(1), 53-60. Retrieved February 12, 2013, from http://arrow.dit.ie/cgi/viewcontent.cgi?article= $1001 \&$ context=buschmanart

Kaiser, F. G., \& Wilson, M. (2004). Goal-directed conservation behavior: The specific composition of a general performance. Personality and Individual Differences, 36(7), 1531-1544. Retrieved June 29, 2012, from http://ac.els-cdn.com/s019188690 3002460/1-s2.0-S0191886903002460-main.pdf? _tid=1b4c81ca-5958-11e4-8a3700000aab0f01\&a cdnat=1413919747_9d64d89f694eb37fdde0 $397055 d d 1$ ac5
Kals, E., Schumacher, D., \& Montada, L. (1999). Emotional affinity toward nature as a motivational basis to protect nature. Environment and Behavior, 31(2), 178-202. Retrieved June 29, 2012, from http://eab.sagepub. com/content/31/2/178.full.pdf

Kim, S., \& Feldt, L. S. (2008). A comparison of tests for equality of two or more independent alpha coefficients. Journal of Educational Measurement, 45(2), 179-193. Retrieved February 15, 2013, from http://onlinelibrary.wiley.com/doi/10.1111/j.17453984.2008.00059.x/abstract

Maloney, M. P., \& Ward, M. P. (1973). Ecology: Let's hear from the people: An objective scale for measurement of ecological attitudes and knowledge. American Psychologist, 28(7), 583-586. Retrieved February 15, 2013, from http://psycnet.apa.org/journals/amp/28/7/ 583.pdf

Mayer, F. S., \& Frantz, C. M. (2004). The connectedness to nature scale: A measure of individuals feeling in community with nature. Journal of Environmental Psychology, 24(4), 503-515. Retrieved July 26, 2012, from http://gse.cat.org.uk/downloads/connectedness _to_nature_paper.pdf

Mokken, R. J., \& Lewis, C. (1982). A nonparametric approach to the analysis of dichotomous item scores. Applied Psychological Measurement, 6(4), 417-430. Retrieved February 16, 2013, from http://conservancy. umn.edu/ bitstream/handle/11299/101545/1/v06n 4p417.pdf

Olivos, P., Aragonés, J. I., \& Amérigo, M. (2011). The connectedness to nature scale and its relationship with environmental beliefs and identity. International Journal of Hispanic Psychology, 4(1), 5-20. Retrieved July 25, 2012, from https://investigacion.uclm.es/ documentos/fi_1331812710-olivos.aragones. amerigo.2011.connectedness.nature.sacle.pdf

Pasquali, L. (2003). Psicometria: teoria dos testes na Psicologia e na Educação. Petrópolis: Vozes.

Perrin, J. L., \& Benassi, V. A. (2009). The connectedness to natures scale: A measure of emotional connection to nature? Journal of Environmental Psychology, 29(4), 434-440. Retrieved July 25, 2012, from http://ac.elscdn.com/S0272494409000243/1-s2.0-S027249440 900 0243-main.pdf?_tid=152911ac-595b-11e4-ac 2e-00000aab0f27\&acdnat=1413921026_33260d9 7d0ad78ca2185795 cadd91633

Pessoa, V. V. (2011). Análise do conhecimento e das atitudes frente às fontes renováveis de energia: uma contribuição da Psicologia (Tese de Doutorado não-publicada). Universidade Federal da Paraíba, João Pessoa. Recuperado em junho 28, 2012, de http:// www.vvgouveia.net/en/images/Teses/Pessoa_V._S._ 2011.pdf.pdf

Primi, R., Muniz, M., \& Nunes, C. H. S. S. (2009). Definições contemporâneas de validade de testes psicológicos. In C. S. Hutz (Org.), Avanços e polêmicas em 
avaliação psicológica (pp.243-266). São Paulo: Casa do Psicólogo.

R Development Core Team. (2011). R: A language and environment for statistical computing. R Foundation for Statistical Computing, Vienna, Austria. Retrieved February 25, 2013, from http://web.mit.edu/r_v3.0.1/ fullrefman.pdf

Schultz, P. W. (2000). Empathizing with nature: The effects of perspective taking on concern for environmental issues. Journal of Social Issues, 56(3), 391-406. Retrieved July 27, 2012, from http://onlinelibrary. wiley.com/

Schultz, P. W. (2001). The structure of environmental concern: Concern for self, other people, and the biosphere. Journal of Environmental Psychology, 21(4), 327-339. Retrieved July 27, 2012, from http://ac.elscdn.com/S0272494401902270/1-s2.0-5027249440 1902270-main.pdf?_tid=8c031 8b8-595b-11e4-8 1de-00000aabOf02\&acdnat=1413921225_171d685 e57b8c 23f1e46e5e6f50d80c2

Schultz, P. W. (2002). Inclusion with nature: Understanding the Psychology of human-nature interactions. In P.
Schmuck \& P. W. Schultz (Eds.), The Psychology of sustainable development (pp.61-78). New York: Kluwer.

Schultz, P. W., Shriver, C., Tabanico, J. J., \& Khazian, A. M. (2004). Implicit connections with nature. Journal of Environmental Psychology, 24(1), 31-42. Retrieved July 27, 2012, from http://ac.els-cdn.com/S02724 94403000227/1-s2.0-S0272494403000227-main. pdf?_tid=c405b590-595b-11e4-92c3-00000aa b0f27\&acdnat=1413921319_fdb5148694305b47d d $87 \mathrm{fb} 0 \mathrm{c} 302 \mathrm{c} 2179$

Tabachnick, B. G., \& Fidell, L. S. (2013). Using multivariate statistics ( $6^{\text {th }}$ ed.). New York: Allyn \& Bacon.

van der Ark, L. A. (2007). Mokken scale analysis in R. Journal of Statistical Software, 20(11), 1-19. Retrieved February 25, 2013, from http://www.jstatsoft.org/v20/ i11/paper

Recebido: novembro 1, 2013

Versão final: outubro 27, 2014

Aprovado: janeiro 8, 2015 